\title{
Bayesian Prediction Based on Type-I Hybrid Censored Data from a General Class of Distributions
}

\author{
Amr Sadek \\ Department of Mathematics, Faculty of Science, Al-Azhar University, Cairo, Egypt \\ Email address: \\ a_sadek@azhar.edu.eg

\section{To cite this article:} \\ Amr Sadek. Bayesian Prediction Based on Type-I Hybrid Censored Data from a General Class of Distributions. American Journal of \\ Theoretical and Applied Statistics. Vol. 5, No. 4, 2016, pp. 192-201. doi: 10.11648/j.ajtas.20160504.15
}

Received: May 4, 2016; Accepted: May 12, 2016; Published: June 14, 2016

\begin{abstract}
One and two-sample Bayesian prediction intervals based on Type-I hybrid censored for a general class of distribution $1-F(\mathrm{x})=[a h(\mathrm{x})+b]^{c}$ are obtained. For the illustration of the developed results, the inverse Weibull distribution with two unknown parameters and the inverted exponential distribution are used as examples. Using the importance sampling technique and Markov Chain Monte Carlo (MCMC) to compute the approximation predictive survival functions. Finally, a real life data set and a generated data set are used to illustrate the results derived here.
\end{abstract}

Keywords: Bayesian Prediction, Type-I Hybrid Censored, General Class, Markov Chain Monte Carlo, Importance Sampling Technique

\section{Introduction}

Prediction plays an important role in different areas of applied statistics such as medical sciences and reliability analysis. Bayesian prediction have more attention among other issues of prediction. Discussion of the prediction intervals (one-sample and two-sample prediction) for a future sample is valuable in lifetime studies. Bayesian prediction intervals for future observations have been discussed by several authors, including Howlader [1], Geisser [2], Raqab and Nagaraja [3], Al-Hussaini and Jaheen ([4]; [5]), Abdel-Aty et al. [6], Kundu and Howlader [7], Mohie El-Din et al. ([8]; [9]), Shafay and Balakrishnan [10], Mohie El-Din and Shafay [11] and Shafay et al. [12]. In this article, we use a general class of distribution (see; Khan and Abu-Salih [13], Athar and Islam [14]) to derive general procedure for determining the one- and two-sample Bayesian prediction intervals based on Type-I hybrid censored data. In the rest of this section, we derive the likelihood function and the conditional density functions of $X s: n$ given the Type-I hybrid censored data. In Section 2, we derive the one-sample Bayesian predictive survival function and the one-sample Bayesian predictions bounds for the $s-t h(r<s \leq n)$ ordered lifetime from Type-I hybrid censored sample. Furthermore, we derive the two-sample Bayesian predictive survival function and the two sample Bayesian predictions bounds for the $s-t h$ ordered lifetime from a future independent sample. In Section 3, special cases of this general class such as the inverse Weibull distribution when the two parameters are unknown and the inverted exponential distribution are considered as illustrative examples, wherein we adopt the importance sampling technique to compute the approximation predictive survival function in the one-sample case and the Markov Chain Monte Carlo (MCMC) method to compute the approximation predictive survival function in the two-sample case. Finally, some numerical examples are conducted to illustrate the prediction procedures.

Let the general form of distributions be

$$
1-F(x)=[a h(x)+b]^{c}, \quad \alpha \leq x \leq \beta,
$$

where a, b and $\mathrm{c}$ are constants $(\mathrm{a} ; \mathrm{c} \neq 0)$ s.t $F(\alpha)=0, F(\beta)=1$ and $h(x) \equiv h(x ; \underline{\theta})$ is a monotonic and differentiable function of $\mathrm{x}$ in the interval $[\alpha, \beta]$ and the parameter $\underline{\theta} \in \Theta$ may be a real vector, then

$$
f(x)=-\operatorname{cah}^{\prime}(x)[a h(x)+b]^{c-1},
$$

where $h^{\prime}(x)=\frac{d}{d x} h(x)$. The following table gives some distributions with proper choice of $\mathrm{a} ; \mathrm{b}$; $\mathrm{c}$ and $\mathrm{h}(\mathrm{x})$ as examples of the general class. 
Table 1. Some distributions derived from the general class.

\begin{tabular}{|c|c|c|c|c|c|}
\hline Distributions & $\bar{F}(x)$ & $\mathbf{a}$ & b & c & $h(x)$ \\
\hline Exponential & $\begin{array}{l}e^{-\lambda x} \\
x>0, \lambda>0\end{array}$ & 1 & 0 & $\lambda$ & $e^{-x}$ \\
\hline $\begin{array}{l}\text { Generalized } \\
\text { Exponential }\end{array}$ & $\begin{array}{l}1-\left(1-e^{-(x-\mu) \lambda}\right)^{\alpha} \\
x>\mu, \lambda>0, \alpha>0\end{array}$ & -1 & 1 & 1 & $\left(1-e^{-(x-\mu) \lambda}\right)^{\alpha}$ \\
\hline $\begin{array}{l}\text { Generalized } \\
\text { Inverted } \\
\text { Exponential }\end{array}$ & $\begin{array}{l}\left(1-e^{-(x-\mu) \lambda}\right)^{\alpha} \\
x>\mu, \lambda>0, \alpha>0\end{array}$ & -1 & 1 & $\alpha$ & $e^{-(x-\mu) \lambda}$ \\
\hline Weibull & $\begin{array}{l}e^{-\theta(x-\mu)^{p}} \\
x \geq \mu\end{array}$ & 1 & 0 & $\theta$ & $e^{-(x-\mu)^{p}}$ \\
\hline Inverse Weibull & $\begin{array}{l}1-e^{-\theta x^{-p}} \\
0 \leq x<\infty\end{array}$ & -1 & 1 & 1 & $e^{-\theta x^{-p}}$ \\
\hline Pareto & $\begin{array}{l}\alpha^{p} x^{-p} \\
\alpha \leq x<\infty\end{array}$ & $\alpha$ & 0 & $p$ & $x^{-1}$ \\
\hline Gumbel & $\begin{array}{l}1-e^{-e^{(x-\mu) \beta}} \\
\beta>0\end{array}$ & -1 & 1 & 1 & $e^{-e^{(x-\mu) \beta}}$ \\
\hline Burr type XII & $\begin{array}{l}\left(1+x^{c}\right)^{-k} \\
x>0, c>0, k>0\end{array}$ & 1 & 1 & $-k$ & $x^{c}$ \\
\hline $\begin{array}{l}\text { Beta of the } \\
\text { first kind }\end{array}$ & $\begin{array}{l}(1-x)^{p} \\
0<x<1\end{array}$ & -1 & 1 & $p$ & $x$ \\
\hline
\end{tabular}

Let $X_{1: n}<X_{2: n}<\cdots<X_{n: n}$ be the order statistics from a random sample of size $n$ from a distribution function $F(x)$ given in (1) with density function $f(x)$ given in (2).

Let $K$ denote the number of $X_{i: n}$ 's that are at most $T$. Then $K$ is a discrete random variable with support $\{0,1, \cdots, n\}$ and probability density function as

$$
P(K=k)=\left(\begin{array}{l}
n \\
k
\end{array}\right) p^{k} q^{n-k}, k=0,1, \cdots, n,
$$

where $p=F(T)$ and $q=1-p=1-F(T)$.

We have one of the two following types of observations:

Case $\quad$ I. $\quad X_{1: n}<X_{2: n}<\cdots<X_{r: n} \quad$ if $\quad X_{r: n} \leq T \quad$ with $r \leq K \leq n$;

Case II: $\quad X_{1: n}<X_{2: n}<\cdots<X_{K: n} \quad$ if $\quad T<X_{r: n} \quad$ with $0 \leq K \leq r-1$.

The likelihood function of a Type-I hybrid censored sample is as follows:

Case I.

$$
L_{1}\left(\theta ; X_{r}\right)=\frac{n !}{(n-r) !} \prod_{i=1}^{r}-c a h^{\prime}\left(x_{i}\right)\left[a h\left(x_{i}\right)+b\right]^{-1}\left[a h\left(x_{r}\right)+b\right]^{(n-r)},
$$

where $X_{r}=\left(x_{1}, \cdots, x_{r}\right)$ and $x_{1}<\cdots<x_{r} \leq T$.

Case II:

$$
L_{2}\left(\theta ; X_{k}\right)=\frac{n !}{(n-k) !} \prod_{i=1}^{k}-c a h^{\prime}\left(x_{i}\right)\left[a h\left(x_{i}\right)+b\right]^{c-1}[a h(T)+b]^{c(n-k)}
$$

where $X_{k}=\left(x_{1}, \cdots, x_{k}\right)$ and $x_{1}<\cdots<x_{k} \leq T<x_{k+1}$.

When $r<s \leq n$, the conditional density function of $X_{s: n}$, given the Type-I hybrid censored data, is obtained as follows:

Case I.

$$
f_{1}\left(x_{s} \mid X_{r}\right)=\left\{\begin{array}{lc}
f_{11}\left(x_{s} \mid X_{r}\right), & x_{r}<x_{s} \leq T, \\
f_{12}\left(x_{s} \mid X_{r}\right), & x_{s}>T,
\end{array}\right.
$$

where

$$
\begin{aligned}
f_{11}\left(x_{s} \mid X_{r}\right) & =\frac{1}{P(r \leq K \leq n)} \sum_{k=s}^{n} f\left(x_{s} \mid x_{r}, K=k\right) P(K=k) \\
& =\sum_{k=s}^{n} \frac{(k-r) ! P(K=k)}{(s-r-1) !(k-s) ! \sum_{j=r}^{n} P(K=j)} \frac{\left[F\left(x_{s}\right)-F\left(x_{r}\right)\right]^{s-r-1}\left[F(T)-F\left(x_{s}\right)\right]^{k-s} f\left(x_{s}\right)}{\left[F(T)-F\left(x_{r}\right)\right]^{k-r}} \\
& =\sum_{k=s}^{n} \sum_{q=0}^{s-r-1} \sum_{w=0}^{k-s} \frac{A_{1} \varphi\left(x_{r}, T\right)\left[a h\left(x_{r}\right)+b\right]^{c(s-r-1-q)}}{\left[\left(a h\left(x_{r}\right)+b\right)^{c}-(a h(T)+b)^{c}\right]^{k-r}} \operatorname{cah}^{\prime}\left(x_{s}\right)\left[a h\left(x_{s}\right)+b\right]^{c^{(q+k-s-w+1)-1}}[a h(T)+b]^{w w},
\end{aligned}
$$

and

$$
\begin{aligned}
f_{12}\left(x_{s} \mid X_{r}\right)= & \frac{1}{P(r \leq K \leq n)} \sum_{k=r}^{s-1} f\left(x_{s} \mid x_{r}, K=k\right) P(K=k) \\
& =\sum_{k=r}^{s-1} \frac{(n-k) ! P(K=k)}{(s-k-1) !(n-s) ! \sum_{j=r}^{n} P(K=j)} \frac{\left[F\left(x_{s}\right)-F(T)\right]^{s-k-1}\left[1-F\left(x_{s}\right)\right]^{n-s} f\left(x_{s}\right)}{[1-F(T)]^{n-k}} \\
& =\sum_{k=r}^{s-1} \sum_{q=0}^{s-k-1} A_{2} \varphi\left(x_{r}, T\right) c a h^{\prime}\left(x_{s}\right)\left[a h\left(x_{s}\right)+b\right]^{c(q+n-s+1)-1}[a h(T)+b]^{c(s-q-n-1)},
\end{aligned}
$$

with 


$$
\begin{gathered}
A_{1}=\frac{(-1)^{q+w+1}(k-r) !}{q ! w !(s-r-1-q) !(k-s-w) !} \\
A_{2}=\frac{(-1)^{q+1}(n-k) !}{q !(n-s) !(s-k-1-q) !}
\end{gathered}
$$

and

$$
\varphi\left(x_{r}, T\right)=\frac{\sum_{i=0}^{k}\left(\begin{array}{l}
n \\
k
\end{array}\right)\left(\begin{array}{l}
k \\
i
\end{array}\right)(-1)^{i}[a(T)+b]^{c(i+n-k)}}{\sum_{j=r}^{n} \sum_{u=0}^{j}\left(\begin{array}{l}
n \\
j
\end{array}\right)\left(\begin{array}{l}
j \\
u
\end{array}\right)(-1)^{u}[a h(T)+b]^{c(u+n-j)}} .
$$

Case II:

$$
\begin{gathered}
f_{2}\left(x_{s} \mid X_{k}\right)=\frac{1}{P(0 \leq k \leq r-1)} \sum_{k=0}^{r-1} f\left(x_{s} \mid x_{k}, K=k\right) P(K=k) \\
=\sum_{k=0}^{r-1} \frac{(n-k) ! P(K=k)}{(s-k-1) !(n-s) ! \sum_{j=0}^{r-1} P(k=j)} \\
\frac{\left[F\left(x_{s}\right)-F(T)\right]^{s-k-1}\left[1-F\left(x_{s}\right)\right]^{n-s} f\left(x_{s}\right)}{[1-F(T)]^{n-k}}
\end{gathered}
$$

$=\sum_{k=0}^{r-1 s-k-1} \sum_{q=0} A_{2} \psi\left(x_{r}, T\right) c a h^{\prime}\left(x_{s}\right)\left[a h\left(x_{s}\right)+b\right]^{(q+n-s+1)-1}[a h(T)+b]^{(s-q-n-1)}$,

where

$$
\psi\left(x_{r}, T\right)=\frac{\sum_{i=0}^{n}\left(\begin{array}{l}
n \\
k
\end{array}\right)\left(\begin{array}{l}
k \\
i
\end{array}\right)(-1)^{i}[a h(T)+b]^{c(i+n-k)}}{\sum_{j=0}^{r-1} \sum_{u=0}^{n j}\left(\begin{array}{l}
n \\
j
\end{array}\right)\left(\begin{array}{l}
j \\
u
\end{array}\right)(-1)^{u}[a h(T)+b]^{c(u+n-j)}} .
$$

\section{Bayesian Analysis}

Bayesian approach has received a lot of attention for estimating the parameters of statistical distributions and for predicting samples. It makes use of ones prior knowledge about the parameters and also takes into consideration the data available. If ones prior knowledge about the parameter is available, it is suitable to make use of an informative prior but in a situation where one does not have any prior knowledge about the parameter and cannot obtain vital information from experts to this regard, then a non-informative prior will be a suitable alternative to use, Guure et al. [15].

Let the prior distribution denoted by $\pi(\theta ; \delta)$, where $\theta \in \Theta$ is the vector of parameters of the distribution under consideration and $\delta$ is the vector of prior parameters. Then the posterior density function of $\theta$, can be written as:

Case I.

$$
\pi_{1}^{*}\left(\theta \mid X_{r}\right)=C_{1}^{-1} L_{1}\left(\theta ; X_{r}\right) \pi(\theta ; \delta),
$$

where $\quad X_{r}=\left(x_{1}, \cdots, x_{r}\right) \quad$ and $\quad x_{1}<\cdots<x_{r} \leq T ; \quad$ and $C_{1}=\int_{\theta} L_{1}\left(\theta ; X_{r}\right) \pi(\theta ; \delta) d \theta$.

Case II.

$$
\pi_{2}^{*}\left(\theta \mid X_{k}\right)=C_{2}^{-1} L_{1}\left(\theta ; X_{k}\right) \pi(\theta ; \delta)
$$

where $X_{k}=\left(x_{1}, \cdots, x_{k}\right)$ and $x_{1}<\cdots<x_{k} \leq T<x_{k+1}$, and $C_{2}=\int_{\theta} L_{2}\left(\theta ; X_{k}\right) \pi(\theta ; \delta) d \theta$

\subsection{One-Sample Bayesian Prediction Intervals}

We simply obtain the predictive survival function of $X_{s: n}$ as follows:

Case I.

$$
\bar{F}_{1}^{*}\left(x_{s} \mid X_{r}\right)=\left\{\begin{array}{lc}
\bar{F}_{11}^{*}\left(x_{s} \mid X_{r}\right), & x_{r}<x_{s} \leq T, \\
\bar{F}_{12}^{*}\left(x_{s} \mid X_{r}\right), & x_{s}>T,
\end{array}\right.
$$

where

$$
\bar{F}_{11}^{*}\left(t \mid X_{r}\right)=\int_{t}^{T} \int_{\theta \in \Theta} f_{11}\left(x_{s} \mid X_{r}\right) \pi_{1}^{*}\left(\theta \mid x_{r}\right) d \theta d x_{s}+\int_{T}^{\beta} \int_{\theta \in \Theta} f_{12}\left(x_{s} \mid X_{r}\right) \pi_{1}^{*}\left(\theta \mid x_{r}\right) d \theta d x_{s},
$$

and

$$
\bar{F}_{12}^{*}\left(t \mid X_{r}\right)=\int_{t}^{\beta} \int_{\theta \in \Theta} f_{12}\left(x_{s} \mid X_{r}\right) \pi_{1}^{*}\left(\theta \mid x_{r}\right) d \theta d x_{s} .
$$

Case II.

$$
\bar{F}_{2}^{*}\left(t \mid X_{k}\right)=\int_{t}^{\beta} \int_{\theta \in \Theta} f_{2}\left(x_{s} \mid X_{r}\right) \pi_{2}^{*}\left(\theta \mid x_{k}\right) d \theta d x_{s} .
$$

The Bayesian predictive $100(1-\gamma) \%$ interval for $X_{s: n}, r<s \leq n$, can be obtained by solving the following two equations:

$$
\bar{F}^{*}\left(L_{X_{s: n}} \mid X\right)=1-\frac{\gamma}{2} \text { and } \bar{F}^{*}\left(U_{X_{s: n}} \mid X\right)=\frac{\gamma}{2}
$$

where

$$
\bar{F}^{*}(t \mid X)= \begin{cases}\bar{F}_{1}^{*}\left(t \mid X_{r}\right), & \text { CaseI }, \\ \bar{F}_{2}^{*}\left(t \mid X_{k}\right), & \text { CaselI }\end{cases}
$$

and $L_{X_{s: n}}$ and $U_{X_{s: n}}$ denote the lower and upper bounds, respectively. 


\subsection{Two-Sample Bayesian Prediction Intervals}

Let us consider a future sample $\left\{Y_{1}, Y_{2}, \cdots, Y_{m}\right\}$ of size $m$, independent of the informative sample $\left\{X_{1}, X_{2}, \cdots, X_{n}\right\}$ and let $Y_{1: m} \leq Y_{2: m} \leq \cdots \leq Y_{m: m}$ be the order statistics of the future sample. Suppose we are interested in the predictive density of the order statistic $Y_{s: m}$ of the future sample, given the informative data set $\left\{X_{1}, X_{2}, \cdots, X_{n}\right\}$. The probability density function of the $s$-th order statistic of the future sample of size $m$ from a continuous distribution with the distribution function $F(x)$ and the probability density function $f(x)$ is given by

$$
f_{Y_{s: m}}(y \mid \theta)=\frac{m !}{(s-1) !(m-s) !}[F(y)]^{s-1}[1-F(y)]^{m-s} f(y),
$$

where $1 \leq s \leq m$; (see, Arnold et al. [16]).

From (1) and (2), we simply obtain the probability density function of the $s$-th order statistic from a general class as follows:

$$
f_{Y_{s: m}}(y \mid \theta)=\sum_{l=0}^{s-1} C_{l} \operatorname{cah}^{\prime}(y)(a h(y)+b)^{c(m+l-s+1)-1},
$$

where $C_{l}=\frac{(-1)^{l+1} m !}{l !(m-s) !(s-l-1) !}$, and we simply obtain the Bayesian predictive density function of $Y_{s: m}$ as follows:

Case I.

$$
f_{1 Y_{S: m}^{*}}^{*}\left(y \mid X_{r}\right)=\sum_{l=0}^{s-1} C_{l} \int_{\theta} c a h^{\prime}(y)(a h(y)+b)^{c(m+l-s+1)-1} \pi_{1}^{*}\left(\theta \mid X_{r}\right) d \theta,
$$

Case II.

$$
f_{2 Y_{s: m}}^{*}\left(y \mid X_{k}\right)=\sum_{l=0}^{s-1} C_{l} \int_{\theta} c a h^{\prime}(y)(a h(y)+b)^{c(m+l-s+1)-1} \pi_{2}^{*}\left(\theta \mid X_{k}\right) d \theta .
$$

From (17) and (18), we simply obtain the predictive survival function of $Y_{s: m}$ as follows:

Case I.

$$
\begin{gathered}
\bar{F}_{1 Y_{s: m}}^{*}\left(t \mid X_{r}\right)=\int_{t}^{\beta} f_{1 Y_{s: m}^{*}}^{*}\left(y \mid X_{r}\right) d y \\
=\sum_{l=0}^{s-1} \frac{C_{l}}{(m+l-s+1)} \int_{\theta}(a h(t)+b)^{c(m+l-s+1)} \pi_{1}^{*}\left(\theta \mid X_{r}\right) d \theta .
\end{gathered}
$$

Case II.

$$
\begin{gathered}
\bar{F}_{2 Y_{s: m}}^{*}\left(t \mid X_{k}\right)=\int_{t}^{\beta} f_{2 Y_{s: m}}^{*}\left(y \mid X_{k}\right) d y \\
=\sum_{l=0}^{s-1} \frac{C_{l}}{(m+l-s+1)} \int_{\theta}(a h(t)+b)^{c(m+l-s+1)} \pi_{2}^{*}\left(\theta \mid X_{k}\right) d \theta .
\end{gathered}
$$

Then, the Bayesian predictive $100(1-\gamma) \%$ interval for $Y_{s: m}, 1 \leq s \leq m$, can be obtained by solving the following two equations:

$$
\bar{F}^{*}\left(L_{Y_{s: m}} \mid X\right)=1-\frac{\gamma}{2} \text { and } \bar{F}^{*}\left(U_{Y_{s: m}} \mid X\right)=\frac{\gamma}{2},
$$

where

$$
\bar{F}_{Y_{s: m}}^{*}(t \mid X)= \begin{cases}\bar{F}_{1 Y_{s: m}}^{*}\left(t \mid X_{r}\right), & \text { CaseI }, \\ \bar{F}_{2 Y_{s: m}}^{*}\left(t \mid X_{k}\right), & \text { CaseII },\end{cases}
$$

and $L_{Y_{s: m}}$ and $U_{Y_{s: m}}$ denote the lower and upper bounds, respectively.

\section{Examples}

In this section, we discuss the Bayesian prediction of observations from the inverse Weibull distribution when both parameters are unknown and from the inverted exponential distribution. To our knowledge, no one study these distributions for determining the Bayesian prediction intervals for future lifetimes based on an observed Type-I hybrid censored data.

\subsection{Inverse Weibull Distribution}

In this subsection, we take a special case from this general class, the inverse Weibull distribution, when $h(x)=e^{-\theta x^{-p}}, a=-1, b=1, \quad$ and $c=1$, we provide the posterior density function depend on the maximum likelihood distribution given in (4) and (5). Here, we assumed that the model parameters $\theta$ and $p$ follow the independent gamma prior density of the following forms:

$$
\begin{gathered}
g_{1}^{*}\left(\theta \mid u_{1}, v_{1}\right) \propto \theta^{u_{1}-1} e^{-v_{1} \theta}, \quad \theta>0, \\
g_{2}^{*}\left(p \mid u_{2}, v_{2}\right) \propto p^{u_{2}-1} e^{-v_{2} p}, \quad p>0,
\end{gathered}
$$

where $u_{1}, v_{1}, u_{2}$, and $v_{2}$ are the hyper-parameters. Then, the joint posterior density function of $\theta$ and $p$, given the Type-I hybrid censored data, can be written as:

Case I.

$$
\pi_{1}^{*}\left(\theta, p \mid x_{r}\right) \propto \theta^{r+u_{1}-1} e^{-\theta\left(\sum_{i=1}^{r} x_{i}^{-p}+v_{1}\right)} p^{r+u_{2}-1}\left[\prod_{i=1}^{r} x_{i}^{-p-1}\right] e^{-v_{2} p}\left[1-e^{-\theta x_{r}^{-p}}\right]^{n-r},
$$

where $X_{r}=\left(x_{1}, \cdots, x_{r}\right)$ and $x_{1}<\cdots<x_{r} \leq T$.

Therefore, the posterior density function of $\theta$ and $p$ given $X_{r}$ can be written as

$$
\pi_{1}^{*}\left(\theta, p \mid x_{r}\right) \propto \pi_{1 \theta}\left(\theta \mid p, X_{r}\right) \pi_{1 p}\left(p \mid X_{r}\right) h_{1}\left(\theta, p \mid X_{r}\right),
$$

where $\pi_{1 \theta}\left(\theta \mid p, X_{r}\right)$ is a gamma density function with the shape and scale parameters as $r+u_{1}$ and $\left(\sum_{i=1}^{r} x_{i}^{-p}+v_{1}\right)$, respectively, $\pi_{1 p}\left(p \mid X_{r}\right)$ is a proper density function given by 


$$
\pi_{1 p}\left(p \mid X_{r}\right) \propto p^{r+u_{2}-1}\left[\prod_{i=1}^{r} x_{i}^{-p-1}\right] e^{-v_{2} p}\left[\sum_{i=1}^{r} x_{i}^{-p}+v_{1}\right]^{-r-u_{1}}
$$

and

$$
h_{1}\left(\theta, p \mid X_{r}\right)=\left[1-e^{-\theta x_{r}^{-p}}\right]^{n-r} .
$$

Case II.

$$
\pi_{2}^{*}\left(\theta, p \mid X_{k}\right) \propto \theta^{k+u_{1}-1} e^{-\theta\left(\sum_{i=1}^{k} x_{i}^{-p}+v_{1}\right)} p^{k+u_{2}-1}\left[\prod_{i=1}^{k} x_{i}^{-p-1}\right] e^{-v_{2} p}\left[1-e^{-\theta T^{-p}}\right]^{n-k}
$$

where $X_{k}=\left(x_{1}, \cdots, x_{k}\right)$ and $x_{1}<\cdots<x_{k} \leq T<x_{k+1}$.

Similarly as above, we can write the posterior density function of $\theta$ and $p$ given $X_{k}$ as

$$
\pi_{2}^{*}\left(\theta, p \mid x_{k}\right) \propto \pi_{2 \theta}\left(\theta \mid p, X_{k}\right) \pi_{2 p}\left(p \mid X_{k}\right) h_{2}\left(\theta, p \mid X_{k}\right)
$$

where $\pi_{2 \theta}\left(\theta \mid p, X_{k}\right)$ is a gamma density function with the shape and scale parameters as $k+u_{1}$ and $\left(\sum_{i=1}^{k} x_{i}^{-p}+v_{1}\right)$, respectively, $\pi_{2 p}\left(p \mid X_{r}\right)$ is proper density function given by

$$
\pi_{2 p}\left(p \mid X_{k}\right) \propto p^{r+u_{2}-1}\left[\prod_{i=1}^{k} x_{i}^{-p-1}\right] e^{-v_{2} p}\left[\sum_{i=1}^{k} x_{i}^{-p}+v_{1}\right]^{-k-u_{1}},
$$

and

$$
h_{2}\left(\theta, p \mid X_{k}\right)=\left[1-e^{-\theta T^{-p}}\right]^{n-k}
$$

\subsubsection{One-Sample Bayesian Prediction}

The conditional density function of $X_{s: n}$ given the Type-I hybrid censored data, (from Eqs (7), (8), (9)), can be written as:

Case I.

$$
\begin{gathered}
f_{11}\left(x_{s} \mid X_{r}\right)=\sum_{k=s}^{n} \frac{(k-r) ! B_{1}(T)}{(s-r-1) !(k-s) !}\left(e^{-\theta x_{s}^{-p}}-e^{-\theta x_{r}^{-p}}\right)^{s-r-1} p \theta x_{s}^{-p-1} \\
e^{-\theta x_{s}^{-p}}\left(e^{-\theta T^{-p}}-e^{-\theta x_{s}^{-p}}\right)^{k-s}\left(e^{-\theta T^{-p}}-e^{-\theta x_{r}^{-p}}\right)^{r-k} \\
=\sum_{k=s}^{n} \sum_{q=0}^{s-r-1} \sum_{w=0}^{k-s} \frac{(-1)^{q+w}(k-r) ! B_{1}(T)\left(e^{-\theta T^{-p}}-e^{-\theta x_{r}-p}\right)^{r-k}}{(s-r-q-1) ! q !(k-s-w) ! w !} \\
\left(e^{-\theta x_{r}^{-p}}\right)^{q}\left(e^{-\theta T^{-p}}\right)^{k-s-w} p \theta x_{s}^{-p-1}\left(e^{-\theta x_{s}^{-p}}\right)^{w+s-r-q}, \quad \text { (30) }
\end{gathered}
$$

where

$$
B_{1}(T)=\frac{\left(\begin{array}{l}
n \\
k
\end{array}\right)\left(e^{-\theta T^{-p}}\right)^{k}\left(1-e^{-\theta T^{-p}}\right)^{n-k}}{\sum_{j=r}^{n}\left(\begin{array}{l}
n \\
j
\end{array}\right)\left(e^{-\theta T^{-p}}\right)^{j}\left(1-e^{-\theta T^{-p}}\right)^{n-j}} .
$$

Similarly, From Eq. (8), we can obtain

$$
\begin{aligned}
f_{12}\left(x_{s} \mid X_{r}\right) & =\sum_{k=r}^{s-1} \frac{(n-k) ! B_{1}(T)}{(s-k-1) !(n-s) !}\left(e^{-\theta x_{s}^{-p}}-e^{-\theta T^{-p}}\right)^{s-k-1}\left(1-e^{-\theta x_{s}^{-p}}\right)^{n-s} p \theta x_{s}^{-p-1} e^{-\theta x_{s}-p}\left(1-e^{-\theta T^{-p}}\right)^{k-n} \\
& =\sum_{k=r}^{s-1} \sum_{l=0}^{s-k-1} \sum_{z=0}^{n-s} \frac{(-1)^{l+z}(n-k) ! B_{1}(T)\left(1-e^{-\theta T^{-p}}\right)^{k-n}}{l !(s-k-l-1) ! z !(n-s-z) !}\left(e^{-\theta T^{-p}}\right)^{l} p \theta x_{s}^{-p-1}\left(e^{-\theta x_{s}^{-p}}\right)^{s-k-l+z} .
\end{aligned}
$$

Case II.

The conditional density function of $X_{s: n}$ given the Type-I hybrid censored data, in this case, can be written as:

$$
\begin{gathered}
f_{2}\left(x_{s} \mid X_{r}\right)=\sum_{k=0}^{r-1} \frac{(n-k) ! B_{2}(T)}{(s-k-1) !(n-s) !}\left(e^{-\theta x_{s}^{-p}}-e^{-\theta T^{-p}}\right)^{s-k-1}\left(1-e^{-\theta x_{s}^{-p}}\right)^{n-s} p \theta x_{s}^{-p-1} e^{-\theta x_{s}^{-p}}\left(1-e^{-\theta T^{-p}}\right)^{k-n} \\
=\sum_{k=0}^{r-1} \sum_{w=0}^{s-k-1} \sum_{u=0}^{n-s} \frac{(-1)^{w+u}(n-k) ! B_{2}(T)\left(1-e^{-\theta T^{-p}}\right)^{k-n}}{(s-k-w-1) ! w ! u !(n-s-u) !}\left(e^{-\theta T^{-p}}\right)^{w} p \theta x_{s}^{-p-1}\left(e^{-\theta x_{s}^{-p}}\right)^{s-k-w+u}
\end{gathered}
$$

where

$$
B_{2}(T)=\frac{\left(\begin{array}{l}
n \\
k
\end{array}\right)\left(e^{-\theta T^{-p}}\right)^{k}\left(1-e^{-\theta T^{-p}}\right)^{n-k}}{\sum_{j=0}^{r-1}\left(\begin{array}{l}
n \\
j
\end{array}\right)\left(e^{-\theta T^{-p}}\right)^{j}\left(1-e^{-\theta T^{-p}}\right)^{n-j}} .
$$

Then, we obtain the predictive survival function of $X_{s: n}$ as follows:

$$
\bar{F}_{11}^{*}\left(t \mid X_{r}\right)=\int_{0}^{\infty} \int_{0}^{\infty}\left(\int_{t}^{T} f_{11}\left(x_{s} ; \theta, p\right) d x_{s}\right) \pi_{1}^{*}\left(\theta, p \mid X_{r}\right) d \theta d p+\int_{0}^{\infty} \int_{0}^{\infty}\left(\int_{T}^{\infty} f_{12}\left(x_{s} ; \theta, p\right) d x_{s}\right) \pi_{1}^{*}\left(\theta, p \mid X_{r}\right) d \theta d p
$$




$$
\begin{gathered}
=\int_{0}^{\infty} \int_{0}^{\infty} \sum_{k=s}^{n} \sum_{q=0}^{s-r-1} \sum_{w=0}^{k-s} \frac{(-1)^{q+w}(k-r) ! B_{1}(T)}{q ! w !(s-r-q-1) !(k-s-w) !} \frac{\left(e^{-\theta T^{-p}}-e^{-\theta x_{r}-p}\right)^{r-k}}{w+s-r-q}\left(e^{-\theta x_{r}-p}\right)^{q}\left(e^{-\theta T^{-p}}\right)^{k-s-w} \\
{\left[\left(e^{-\theta T^{-p}}\right)^{w+s-r-q}-\left(e^{-\theta t^{-p}}\right)^{w+s-r-q}\right] \pi_{1}^{*}\left(\theta, p \mid X_{r}\right) d \theta d p} \\
+\int_{0}^{\infty} \int_{0}^{\infty} \sum_{k=r}^{s-1} \sum_{l=0}^{s-k-1 n-s} \sum_{z=0} \frac{(-1)^{l+z}(n-k) !(s-k-1) ! B_{1}(T)}{l ! z !(s-r-1) !(s-k-l-1) !} \frac{\left(1-e^{-\theta T^{-p}}\right)^{k-n}}{(s-k-l+z)(n-s-z) !}\left(e^{-\theta T^{-p}}\right)^{l} \\
{\left[1-\left(e^{-\theta T^{-p}}\right)^{s-k-l+z}\right] \pi_{1}^{*}\left(\theta, p \mid X_{r}\right) d \theta d p .}
\end{gathered}
$$

and similarly

$$
\begin{aligned}
& \bar{F}_{12}^{*}\left(t \mid X_{r}\right)=\int_{t}^{\infty} \int_{0}^{\infty} \int_{0}^{\infty} f_{12}\left(x_{s} \mid X_{r}\right) \pi_{1}^{*}\left(\theta, p \mid X_{r}\right) d \theta d p d x_{s} \\
&=\int_{0}^{\infty} \int_{0}^{\infty} \sum_{k=r}^{s-1} \sum_{l=0}^{s-k-1 n-s} \sum_{z=0}^{n-s} \frac{(-1)^{l+z}(n-k) ! B_{1}(T)}{l !(s-k-l-1) ! z !} \frac{\left(1-e^{-\theta T^{-p}}\right)^{k-n}}{(n-s-z) !(s-k-l+z)}\left(e^{-\theta T^{-p}}\right)^{l} \\
&\left(1-e^{-\theta t^{-p}}\right)^{s-k-l+z} \pi_{1}^{*}\left(\theta, p \mid x_{r}\right) d \theta d p . \\
& \bar{F}_{2}^{*}\left(t \mid X_{k}\right)= \int_{t}^{\infty} \int_{0}^{\infty} \int_{0}^{\infty} f_{2}\left(x_{s} \mid X_{k}\right) \pi_{2}^{*}\left(\theta, p \mid X_{k}\right) d \theta d p d x_{s} \\
&= \int_{0}^{\infty} \int_{0}^{\infty} \sum_{k=0}^{\infty} \sum_{w=0}^{r-1} \sum_{u=0}^{s-k-1 n-s} \frac{(-1)^{w+u}(n-k) ! B_{2}(T)}{(s-k-w-1) ! w ! u !} \frac{\left(1-e^{-\theta T^{-p}}\right)^{k-n}}{(n-s-u) !(s-k-w+u)}\left(e^{-\theta T^{-p}}\right)^{w} \\
&\left(1-e^{-\theta t^{-p}}\right)^{s-k-w+u} \pi_{2}^{*}\left(\theta, p \mid x_{k}\right) d \theta d p .
\end{aligned}
$$

It does not seem to be possible to compute the probabilities in Eqs (33), (34), (35) analytically. Hence, we use the importance sampling technique, (see, Geweke [17]; Chen and Shao ([18]; [19])) to construct the Bayesian prediction interval. The details are explained below.

\section{Importance Sampling technique}

Firstly, we need to prove that the $\pi_{p}(p \mid$ data $)$ as given in (24) and (28) has a log-concave density function: From (24), the $\ln \pi_{1 p}\left(p \mid X_{r}\right)$ without the additive constant is

$$
\begin{aligned}
\ln \pi_{p}\left(p \mid X_{r}\right) & =\ln c+\left(r+u_{2}-1\right) \ln p \\
& -v_{2} p-(p+1) \sum_{i=1}^{r} \ln x_{i}-\left(r+u_{1}\right) \ln \left[\sum_{i=1}^{r} x_{i}^{-p}+v_{1}\right]
\end{aligned}
$$

it is easy to show that $\frac{d^{2}}{d p^{2}} \ln \pi_{1 p}\left(p \mid X_{r}\right)<0$, which implies that $\pi_{1 p}(p \mid$ data $)$ has a log-concave density function.

Since $\pi_{1 p}(p \mid$ data $)$ has a log-concave density, using the idea of Devroye [20], it is possible to generate sample from $\pi_{1 p}(p \mid$ data $)$. Moreover, since $\pi_{1 \theta}(\theta \mid p$, data $)$ follows gamma, it is quite simple to generate sample from $\pi_{1 \theta}(\theta \mid p$, data $)$. Now we would like to provide the importance sampling procedure to compute the probabilities in Eqs (33), (34), (35).

Algorithm:
- Step1: Generate $p$ from $\pi_{1 p}(p \mid$ data $)$ using the method developed by Devroye [20].

- Step2: Generate $\theta$ from $\pi_{1 \theta}(\theta \mid p$, data $)$.

- Step3: Repeat Step 1 and Step 2 and obtain $\left(p_{1} ; \theta_{1}\right),\left(p_{2} ; \theta_{2}\right), \cdots,\left(p_{M} ; \theta_{M}\right)$.

- Step4: The approximate value of $\int_{0}^{\infty} \int_{0}^{\infty} f(\theta, p) \pi_{1}^{*}\left(\theta, p \mid X_{r}\right) d \theta d p$ can be obtained as

$$
\int_{0}^{\infty} \int_{0}^{\infty} f(\theta, p) \pi_{1}^{*}\left(\theta, p \mid X_{r}\right) d \theta d p=\frac{\frac{1}{M} \sum_{i=1}^{M} f\left(\theta_{i}, p_{i}\right) h_{1}\left(\theta_{i}, p_{i} \mid X_{r}\right)}{\frac{1}{M} \sum_{i=1}^{M} h_{1}\left(\theta_{i}, p_{i} \mid X_{r}\right)} .
$$

Similary, we can use the above algorithm to compute $\int_{0}^{\infty} \int_{0}^{\infty} f(\theta, p) \pi_{2}^{*}\left(\theta, p \mid X_{k}\right) d \theta d p$.

The Bayesian predictive $100(1-\gamma) \%$ interval for $X_{s: n} ; r<s \leq n$; can be obtained by solving the two equations given in (16).

\subsubsection{Two-Sample Bayesian Prediction}

The predictive survival function of $Y_{s: m}$ in this special case is obtained as follows:

Case I.

$$
\bar{F}_{1 Y_{s: m}}^{*}\left(t \mid X_{r}\right)=\int_{t}^{\infty} f_{1 Y_{s: m}}^{*}\left(y \mid X_{r}\right) d y
$$




$$
=\sum_{l=0}^{s-1} \frac{C_{l}}{(m+l-s+1)} \int_{0}^{\infty} \int_{0}^{\infty}\left(1-e^{-\theta t^{-p}}\right)^{m+l-s+1} \pi_{1}^{*}\left(\theta, p \mid X_{r}\right) d \theta d p .
$$

Case II.

$$
\begin{gathered}
\bar{F}_{2 Y_{s: m}}^{*}\left(t \mid X_{k}\right)=\int_{t}^{\infty} f_{2 Y_{s: m}}^{*}\left(y \mid X_{k}\right) d y \\
=\sum_{l=0}^{s-1} \frac{C_{l}}{(m+l-s+1)} \int_{0}^{\infty} \int_{0}^{\infty}\left(1-e^{-\theta t^{-p}}\right)^{m+l-s+1} \pi_{2}^{*}\left(\theta, p \mid X_{k}\right) d \theta d p,
\end{gathered}
$$

where $C_{l}=\frac{(-1)^{l+1} m !}{l !(m-s) !(s-l-1) !}$.

By the same importance sample technique, the Bayesian predictive $100(1-\gamma) \%$ interval for $Y_{s: m}, 1 \leq s \leq m$, can be obtained by solving the two equations in (21).

\subsection{Inverted Exponential Distribution}

The inverted exponential distribution is a special case from inverse Weibull distribution when the shape parameter is known $(p=1)$. we provide the posterior density function depend on the maximum likelihood distribution given in (4) and (5), when the shape parameter is $p=1$. It is assumed that the scale parameter has a gamma prior distribution with the shape and scale parameters as $u$ and $v$, respectively and it has the probability density function

$$
\pi(\theta \mid u, v) \propto \theta^{u-1} e^{-v \theta}, \quad \theta>0 .
$$

The posterior density function of $\theta$, given the Type-I hybrid censored data, can be written as:

Case I.

$$
\pi_{1}^{*}\left(\theta \mid x_{r}\right)=\frac{\sum_{j=0}^{n-r}\left(\begin{array}{c}
n-r \\
j
\end{array}\right)(-1)^{j} \theta^{r+u-1} e^{-\theta\left(\frac{j}{x_{r}}+\sum_{i=1}^{r} \frac{1}{x_{i}}+v\right)}}{\sum_{j=0}^{n-r}\left(\begin{array}{c}
n-r \\
j
\end{array}\right)(-1)^{j}\left(\frac{j}{x_{r}}+\sum_{i=1}^{r} \frac{1}{x_{i}}+v\right)^{-(r+u)} \Gamma(r+u)},
$$

where $X_{r}=\left(x_{1}, \cdots, x_{r}\right)$ and $x_{1}<\cdots<x_{r} \leq T$.

Case II.

$$
\pi_{2}^{*}\left(\theta \mid x_{k}\right)=\frac{\sum_{j=0}^{n-k}\left(\begin{array}{c}
n-k \\
j
\end{array}\right)(-1)^{j} \theta^{k+u-1} e^{-\theta\left(\frac{j}{T}+\sum_{i=1}^{k} \frac{1}{x_{i}}+v\right)}}{\sum_{j=0}^{n-k}\left(\begin{array}{c}
n-k \\
j
\end{array}\right)(-1)^{j}\left(\frac{j}{T}+\sum_{i=1}^{k} \frac{1}{x_{i}}+v\right)^{-(k+u)} \Gamma(k+u)}
$$

where $X_{k}=\left(x_{1}, \cdots, x_{k}\right)$ and $x_{1}<\cdots<x_{k} \leq T<x_{k+1}$.

In this case the predictive survival function of $X_{s: n}$ is the same as above when we put $p=1$.

\subsubsection{One-Sample Bayesian Prediction}

To compute $\int_{0}^{\infty} f(\theta) \pi_{1}^{*}\left(\theta \mid X_{r}\right) d \theta$ by using the MCMC technique, we use the following procedure:

- Step 1. Generate $\theta_{1}$ from $\pi_{1}^{*}\left(\theta \mid X_{r}\right)$
- Step 2. Repeat Step 1 and obtain $\theta_{1}, \theta_{2}, \ldots, \theta_{N}$;

- Step 3. The approximate value of $\int_{0}^{\infty} f(\theta) \pi_{1}^{*}\left(\theta \mid X_{r}\right) d \theta$ is then obtained as

$$
\int_{0}^{\infty} f(\theta) \pi_{1}^{*}\left(\theta \mid X_{r}\right) d \theta=\frac{\sum_{i=1}^{N} f\left(\theta_{i}\right)}{N} .
$$

Similarly, we can use the above algorithm to compute $\int_{0}^{\infty} g(\theta) \pi_{2}^{*}\left(\theta \mid X_{k}\right) d \theta$

The Bayesian predictive $100(1-\gamma) \%$ interval for $X_{s: n} ; r<s \leq n$; can be obtained by solving the two equations given in (16).

\subsubsection{Two-Sample Bayesian Prediction}

The predictive survival function of $Y_{s: m}$ in this special case is obtained as follows:

Case I.

$$
\begin{gathered}
\bar{F}_{1 Y_{s: m}}^{*}\left(t \mid X_{r}\right)=\int_{t}^{\infty} f_{1 Y_{s: m}^{*}}^{*}\left(y \mid X_{r}\right) d y \\
=\sum_{l=0}^{s-1} \frac{C_{l}}{(m+l-s+1)} \int_{0}^{\infty}\left(1-e^{-\theta t^{-1}}\right)^{m+l-s+1} \pi_{1}^{*}\left(\theta \mid X_{r}\right) d \theta .
\end{gathered}
$$

Case II.

$$
\begin{gathered}
\bar{F}_{2 Y_{s: m}}^{*}\left(t \mid X_{k}\right)=\int_{t}^{\infty} f_{2 Y_{s: m}}^{*}\left(y \mid X_{k}\right) d y \\
=\sum_{l=0}^{s-1} \frac{C_{l}}{(m+l-s+1)} \int_{0}^{\infty}\left(1-e^{-\theta t^{-1}}\right)^{m+l-s+1} \pi_{2}^{*}\left(\theta \mid X_{k}\right) d \theta .
\end{gathered}
$$

Then, the Bayesian predictive interval for $Y_{s: m}, 1 \leq s \leq m$, can be obtained by the same manner.

\section{Numerical Results}

In this section we consider a real life data set which the inverse Weibull distribution fits it well and another generated data set from the inverted exponential distribution to illustrate the methods proposed in the previous sections.

The real data set is given by Dumonceaux and Antle [21], and it represents the maximum flood levels (in millions of cubic feet per second) of the Susquehenna River at Harrisburg, Pennsylvania over 20 four-year periods (1890-1969) as: 0.654 , $0.613,0.315,0.449,0.297,0.402,0.379,0.423,0.379,0.324$, $0.269,0.740,0.418,0.412,0.494,0.416,0.338,0.392,0.484$, 0.265. Maswadah [22] and Singh et al. [23] checked the suitability of the inverse Weibull distribution to this real data set and concluded that the inverse Weibull distribution fits the data very well.

We consider two different Type-I hybrid censoring schemes:

1. When $n=20, r=15$ and $T=0.43$. In this case, the life-test would have terminated at $T<x_{15: 20}$, and we have obtained the folloing data: $0.265,0.269,0.297,0.315,0.324$, 
$0.338,0.379,0.379,0.392,0.402,0.412,0.416,0.418$ and 0.423 ;

2. When $n=20, r=15$ and $T=0.47$. In this case, the life-test would have terminated at $x_{15: 20}=0.449$, and we have obtained the following data: $0.265,0.269,0.297,0.315,0.324$, $0.338,0.379,0.379,0.392,0.402,0.412,0.416,0.418,0.423$ and 0.449 .

By using the procedures presented earlier, we construct 95\% one-sample Bayesian prediction intervals for order statistics $X_{s: n}, s=16, \cdots, 20$ as well as $95 \%$ two-sample Bayesian prediction intervals for order statistics $Y_{s: m}, s=1,5,10,20$ from a future sample of size $m=20$. To explore the sensitivity of the predictors with respect to the hyperparameters $\left(u_{1}, v_{1}, u_{2}, v_{2}\right)$, we have considered the following four hyperparameters: $(1,7,3,1),(1,7,3,2)$, $(1,8,3,1),(1,8,3,2)$. Table 2 presents the lower and upper 95\% one-sample Bayesian prediction bounds for $X_{s: n}, s=16, \cdots, 20$ for these four choices of the

$\begin{array}{lllllll}0.45175 & 0.84893 & 0.95595 & 1.33698 & 1.38886 & 1.42612 \\ 2.91384 & 3.34748 & 3.39207 & 3.49797 & .41589 & 4.56534 \\ .0716 & 11.7103 & 13.3331 & 78.4651 & 135.999 & 848.432\end{array}$

The corresponding results for the one-sample and two-sample prediction intervals are represented in Tables 4 and 5 , respectively.

\section{Concluding Remarks}

In this paper, we obtained one and two sample prediction bounds based on Type-I hybrid censored data under the general class of distributions. We introduced two examples, the inverse Weibull distribution with unknown two parameters and the inverted exponential distribution, to illustrate the developed results. Bayesian predictive survival function can not be obtained in closed form and so importance sampling technique and Markov Chain Monte Carlo samples, which are then used to compute the approximate predictive survival function. Finally, some numerical results are presented to hyperparameters. Similarly, the lower and upper 95\% two-sample Bayesian prediction bounds for $Y_{s: m}, s=1,5,10,20$ for the different choices of the hyperparameters are presented in Table 3.

Now we generate another data set to illustrate the predictions results for the inverted exponential distribution, we follow the steps

1. given the set of prior parameters, generate the parameter $\theta$,

2. using the generated population parameter, generate an inverted exponential random sample of size $n$,

3. follow the procedures presented in Section 2.2 to construct one-sample and two-sample Bayesian prediction intervals based on Type-I hybrid censored data.

Given the set of prior parameters (let $u=30, v=11$, ), we generated the parameter $\theta$ from prior distribution, $\theta=2.7$ then generated the inverted exponential random sample of size $n=30$, the generated sample is listed as the following:

\section{$\begin{array}{llllll}1.89285 & 2.0541 & .18509 & 2.60002 & 2.64192 & 2.8265\end{array}$ \\ $\begin{array}{llllll}4.67023 & 5.94493 & 8.93186 & 9.01355 & 9.69029 & 10.5754\end{array}$}

illustrate the results and we observe the following remarks:

1. From Tables 2-5, we notice that the lengths of the Bayesian prediction intervals are short when there are a large number of observed values. It is clear that when we use the same value of $r$ but larger value of $T$, the Bayesian prediction intervals become tighter.

2. It is observed that the prediction intervals tend be wider when $\mathrm{s}$ increase. This is a natural, since the prediction of the future order statistic that is far a way from the last observed value has less accuracy than that of other future order statistics.

3. It is evident from Tables 2 and 3 that the lower bounds of Bayesian prediction are relatively insensitive to the specification of the hyperparameters $\left(u_{1}, v_{1}, u_{2}, v_{2}\right)$ while the upper bounds are somewhat sensitive.

Table 2. $95 \%$ one-sample Bayesian prediction bounds for $X_{s: n}, s=16, \cdots, 20$ from inverse Weibull distribution.

\begin{tabular}{|c|c|c|c|c|c|c|c|c|}
\hline \multicolumn{9}{|c|}{$\mathrm{r}=15$ and $\mathrm{T}=0.43$} \\
\hline$\left(u_{1}, v_{1}, u_{2}, v_{2}\right)$ & $(1,7,3,1)$ & & $(1,7,3,2)$ & & $(1,8,3,1)$ & & $(1,8,3,2)$ & \\
\hline$s$ & $L_{X_{s: n}}$ & $U_{X_{s: n}}$ & $L_{X_{s: n}}$ & $U_{X_{s: n}}$ & $L_{X_{s: n}}$ & $U_{X_{s: n}}$ & $L_{X_{s: n}}$ & $U_{X_{s: n}}$ \\
\hline 16 & 0.43907 & 0.63118 & 0.44275 & 0.68220 & 0.44132 & 0.66464 & 0.44615 & 0.79875 \\
\hline 17 & 0.45561 & 0.78217 & 0.45847 & 0.78082 & 0.45652 & 0.82273 & 0.46265 & 0.85957 \\
\hline 18 & 0.47628 & 0.96853 & 0.47105 & 0.81186 & 0.47338 & 0.91154 & 0.48197 & 0.97904 \\
\hline 19 & 0.49904 & 1.19722 & 0.51241 & 1.34441 & 0.49287 & 1.02787 & 0.50513 & 1.17879 \\
\hline 20 & 0.53634 & 1.80118 & 0.55875 & 2.22601 & 0.51996 & 1.14195 & 0.56461 & 2.81921 \\
\hline \multicolumn{9}{|c|}{$\mathrm{r}=15$ and $\mathrm{T}=0.47$} \\
\hline$\left(u_{1}, v_{1}, u_{2}, v_{2}\right)$ & $(1,7,3,1)$ & & $(1,7,3,2)$ & & $(1,8,3,1)$ & & $(1,8,3,2)$ & \\
\hline $\mathbf{S}$ & $L_{X_{s: n}}$ & $U_{X_{s: n}}$ & $L_{X_{s: n}}$ & $U_{X_{s: n}}$ & $L_{X_{s: n}}$ & $U_{X_{s: n}}$ & $L_{X_{s: n}}$ & $U_{X_{s: n}}$ \\
\hline 16 & 0.44205 & 0.53420 & 0.44617 & 0.57326 & 0.44310 & 0.56012 & 0.44717 & 0.59083 \\
\hline 17 & 0.44273 & 0.54657 & 0.45013 & 0.65100 & 0.45077 & 0.65790 & 0.45082 & 0.66844 \\
\hline 19 & 0.46014 & 0.84750 & 0.47350 & 1.10977 & 0.50554 & 0.99213 & 0.47699 & 1.07860 \\
\hline 20 & 0.50359 & 1.70414 & 0.51569 & 1.19332 & 0.51248 & 1.04670 & 0.52939 & 2.33474 \\
\hline
\end{tabular}


Table 3. $95 \%$ two-sample Bayesian prediction bounds for $Y_{s m}, s=1,5,10,15,20 ; m=20$ from inverse Weibull distribution.

\begin{tabular}{|c|c|c|c|c|c|c|c|c|}
\hline \multicolumn{9}{|c|}{$r=15$ and $T=0.43$} \\
\hline \multirow{2}{*}{$\begin{array}{l}\left(u_{1}, v_{1}, u_{2}, v_{2}\right) \\
\mathbf{s}\end{array}$} & \multicolumn{2}{|l|}{$(1,7,3,1)$} & \multicolumn{2}{|l|}{$(1,7,3,2)$} & \multicolumn{2}{|l|}{$(1,8,3,1)$} & \multicolumn{2}{|l|}{$(1,8,3,2)$} \\
\hline & $L_{Y_{s: m}}$ & $U_{Y_{s: m}}$ & $L_{Y_{s: m}}$ & $U_{Y_{s: m}}$ & $L_{Y_{s: m}}$ & $U_{Y_{s: m}}$ & $L_{Y_{s: m}}$ & $U_{Y_{s: m}}$ \\
\hline 1 & 0.20628 & 0.32424 & 0.18440 & 0.31540 & 0.21846 & 0.33201 & 0.18620 & 0.32965 \\
\hline 5 & 0.27808 & 0.40067 & 0.26681 & 0.39713 & 0.28045 & 0.39171 & 0.26439 & 0.39187 \\
\hline 10 & 0.32206 & 0.48557 & 0.32817 & 0.50129 & 0.32436 & 0.46338 & 0.32665 & 0.52512 \\
\hline 15 & 0.37542 & 0.59968 & 0.38940 & 0.71546 & 0.37992 & 0.62889 & 0.38733 & 0.71203 \\
\hline 20 & 0.51570 & 2.12839 & 0.53647 & 2.34615 & 0.49280 & 1.73166 & 0.53828 & 2.52157 \\
\hline \multicolumn{9}{|c|}{$r=15$ and $T=0.47$} \\
\hline$\left(u_{1}, v_{1}, u_{2}, v_{2}\right)$ & $(1,7,3,1)$ & & $(1,7,3,2)$ & & $(1,8,3,1)$ & & $(1,8,3,2)$ & \\
\hline $\mathbf{S}$ & $L_{Y_{s: m}}$ & $U_{Y_{s: m}}$ & $L_{Y_{s: m}}$ & $U_{Y_{s: m}}$ & $L_{Y_{s: m}}$ & $U_{Y_{s: m}}$ & $L_{Y_{s: m}}$ & $U_{Y_{s: m}}$ \\
\hline 1 & 0.21639 & 0.31731 & 0.20012 & 0.32035 & 0.21495 & 0.32431 & 0.19757 & 0.31722 \\
\hline 5 & 0.28039 & 0.38947 & 0.26345 & 0.38254 & 0.27969 & 0.38454 & 0.27278 & 0.39054 \\
\hline 10 & 0.32728 & 0.46743 & 0.32356 & 0.48846 & 0.32377 & 0.46018 & 0.32371 & 0.49926 \\
\hline 15 & 0.37368 & 0.57193 & 0.38515 & 0.66518 & 0.37618 & 0.59236 & 0.38739 & 0.68362 \\
\hline
\end{tabular}

Table 4. $95 \%$ one-sample Bayesian prediction bounds for $X_{s: n}, s=26, \cdots, 30$. from inverted exponential distribution.

\begin{tabular}{lllll}
\hline & \multicolumn{2}{l}{$\mathbf{r}=\mathbf{2 5}$ and $\mathbf{T}=\mathbf{1 0 . 6}$} & \multicolumn{2}{c}{$\mathbf{r}=\mathbf{2 5}$ and $\mathbf{T}=\mathbf{1 1 . 5}$} \\
\hline $\mathbf{S}$ & $\boldsymbol{L}_{\boldsymbol{X}_{\boldsymbol{s} \mathbf{n}}}$ & $\boldsymbol{U}_{\boldsymbol{X}_{\boldsymbol{s} \boldsymbol{n}}}$ & $\boldsymbol{L}_{\boldsymbol{X}_{\text {s:n }}}$ & $\boldsymbol{U}_{\boldsymbol{X}_{\boldsymbol{s}: \boldsymbol{n}}}$ \\
\hline 26 & 11.6371 & 52.0702 & 9.3644 & 20.9758 \\
27 & 13.3179 & 80.5060 & 9.8224 & 36.2551 \\
28 & 15.4181 & 138.939 & 11.3103 & 70.4118 \\
29 & 19.5173 & 371.245 & 12.4387 & 194.071 \\
30 & 28.6091 & 3685.73 & 16.8671 & 2019.12 \\
\hline
\end{tabular}

Table 5. $95 \%$ two-sample Bayesian prediction bounds for $Y_{s: m}, s=1,5,10,20,30 ; m=30$ from inverted exponential distribution.

\begin{tabular}{lllll}
\hline & \multicolumn{2}{c}{$\mathbf{r}=\mathbf{2 5}$ and $\mathbf{T}=\mathbf{1 0 . 6}$} & \multicolumn{2}{c}{$\mathbf{r}=\mathbf{2 5}$ and $\mathbf{T}=\mathbf{1 1 . 5}$} \\
\hline $\mathbf{S}$ & $\boldsymbol{L}_{\boldsymbol{Y}_{\boldsymbol{s}: \boldsymbol{m}}}$ & $\boldsymbol{U}_{\boldsymbol{Y}_{\boldsymbol{s}: \boldsymbol{m}}}$ & $\boldsymbol{L}_{\boldsymbol{Y}_{\boldsymbol{s}: \boldsymbol{m}}}$ & $\boldsymbol{U}_{\boldsymbol{Y}_{\boldsymbol{s} \boldsymbol{m}}}$ \\
\hline 1 & 0.35180 & 1.28963 & 0.34616 & 1.25820 \\
5 & 0.84871 & 2.38517 & 0.82808 & 2.25091 \\
10 & 1.37635 & 3.97899 & 1.36568 & 3.83175 \\
20 & 3.28013 & 12.5604 & 3.22973 & 11.9778 \\
30 & 20.5459 & 3083.74 & 20.1275 & 3016.79 \\
\hline
\end{tabular}

\section{References}

[1] Howlader, H. A., 1985. HPD prediction intervals for Rayleigh distribution. IEEE Trans. Reliab. 34, 121-123.

[2] Geisser, S., 1993. Predictive Inference: An Introduction. Chapman \& Hall, New York.

[3] Raqab, M. Z. and Nagaraja, H. N., 1995. On some predictors of future order statistic. Metron. 53, 185-204.

[4] Al-Hussaini, E. K. and Jaheen, Z. F., 1995. Bayesian prediction bounds for the Burr type XII model. Commun Stat Theory Methods. 24, 1829-1842.

[5] Al-Hussaini, E. K. and Jaheen, Z. F., 1996. Bayesian prediction bounds for the Burr type XII distribution in the presence of outliers. J Stat Plan Inference. 55, 23-37.

[6] Abdel-Aty, Y.; Franz, J., Mahmoud, M. A. W., 2007. Bayesian prediction based on generalized order statistics using multiply Type II censored. Statistics. 41, 495-504.
[7] Kundu, D. and Howlader, H., 2010. Bayesian inference and prediction of the inverse Weibull distribution for Type-II censored data. Comput Stat Data Anal. 54, 1547-1558.

[8] Mohie El-Din, M. M., Abdel-Aty, Y. and Shafay, A. R., 2011. Two sample Bayesian prediction intervals for order statistics based on the inverse exponential-type distributions using right censored sample. J. of the Egy. Math. Societ. 19, 102-105.

[9] Mohie El-Din, M. M., Abdel-Aty, Y. and Shafay, A. R., 2011. Bayesian prediction for order statistics from a general class of distributions based on left Type-II censored data. Int. j. math. comput. 13, 1-8.

[10] Shafay, A. R. and Balakrishnan, N., 2010. One- and two-sample Bayesian prediction intervals based on Type-I hybrid censored data. Commun Stat Simul Comput. 41, 65-88.

[11] Mohie El-Din, M. M. and Shafay, A. R., 2013. One- and two-sample Bayesian prediction intervals based on progressively Type-II censored data. Stat Pap. 54, 287-307.

[12] Shafay, A. R., Balakrishnan, N. and Abdel-Aty, Y., 2014. Bayesian inference based on a jointly Type-II censored sample from two exponential populations. J Stat Comput Simul 84, 2427-2440.

[13] Khan, A. H. and Abu-Salih, M. S., 1989. Characterization of probability distributions by conditional expectation of order statistics. Metron. 47, 171-181.

[14] Athar, H. and Islam, H., 2004. Recurrence relations for single and product moments of generalized order statistics from a general class of distribution, Metron. LXII, 3, 327-337.

[15] Guure, C. B., Ibrahim, N. A. and Al Omari, A. M., 2012. Bayesian estimation of two-parameter Weibull distribution using extension of Jeffreys' prior information with three loss functions, Math Probl Eng. Article ID 589640, 13 pages.

[16] Arnold, B. C., Balakrishnan, N. and Nagaraja, H. N., 1992. A First Course in Order Statistics. New York: John Wiley \& Sons.

[17] Geweke, J., 1989. Bayesian inference in econometrics models using Monte Carlo integration. Econometrica. 57, 1317-1339.

[18] Chen, M.-H. and Shao, Q. M., 1997. On Monte Carlo methods for estimating ratios of normalizing constants. Ann. Statist. 25, 1563-1594. 
[19] Chen, M-H. and Shao, Q. M., 1999. Monte Carlo estimation of Bayesian credible and HPD intervals, J. Comput. Graph. Statist. $8(1), 69-92$.

[20] Devroye, L., 1984. A simple algorithm for generating random variates with a log-concave density function. Computing. 33, 247-257.

[21] Dumonceaux, R. and Antle, C. E., 1973. Discriminating between the log-normal and Weibull distribution. Technometrics. 15, 923-926.
[22] Maswadah, M., 2003. Conditional confidence interval estimation for the inverse Weibull distribution based on censored generalized order statistics. J Stat Comput Simul. 73(12), 887-898.

[23] Singh, S. K., Singh, U. and Sharma, V. K., 2013. Bayesian prediction of future observations from inverse Weibull distribution based on Type-II hybrid censored sample. Int. $j$. adv. stat. probab. 1(2), 32-43. 